\title{
Incubação de ovos e larvas de pacu (Piaractus mesopotamicus holmberg, 1887) em incubadoras do tipo israelense e woynarovich
}

\section{Incubation of eggs and larvae of pacu (Piaractus mesopotamicus holmberg, 1887) in israeli and woynarovich incubators}

\author{
Luis Ricardo Jayme Guerreiro ${ }^{1 *}$; Jeferson Antonio Dias Dias²; Darci Carlos \\ Fornari $^{3}$; Ricardo Pereira Ribeiro ${ }^{4}$; Marco Antonio Zanoni ${ }^{5}$
}

\begin{abstract}
Resumo
O objetivo neste estudo foi avaliar as taxas de fertilização e eclosão dos ovos de Piaractus mesopotamicus induzidos com extrato de hipófise, além da sobrevivência e porcentagem de larvas defeituosas após 48 e 72 horas da eclosão em incubadoras do tipo "Israelense" e "Woynarovich". Utilizou se um "Pool" de ovos e larvas obtidas de 10 fêmeas e 20 machos. O delineamento experimental foi totalmente casualisado com dois tratamentos e quatro repetições. As médias de fertilização não demonstraram diferenças significativas $(\mathrm{P}>0,05)$ entre as incubadoras. Os valores de eclosão $(\%)$ foram significativamente maiores $(\mathrm{P}<0,05)$ nas incubadoras do tipo Woynarovich, a sobrevivência $(\%)$ das larvas após 48 horas da eclosão foi significativamente maior $(\mathrm{P}<0,05)$ nas incubadoras do tipo Israelense, o mesmo foi observado após 72 horas da eclosão. A porcentagem de larvas defeituosas foi significativamente menor $(\mathrm{P}<0,05)$ nas incubadoras do tipo israelense nos dois períodos avaliados. De acordo com os resultados obtidos, as incubadoras do tipo "Israelense" demonstraram melhores condições para a sobrevivência das larvas.
\end{abstract}

Palavras-chave: Desenvolvimento larval. Larvicultura.

\begin{abstract}
The aim of this work was to evaluate the fertilization and hatching rates of eggs of Piaractus mesopotamicus hormonally induced, and the survivorship and percentage of defective larvae at 48 and 72 hours after hatching in Israeli and Woynarovich hatcheries. A pool of eggs and larvae obtained from ten females and twenty males was used. The experimental design was completely randomized with two treatments and four replicates. The fertilization averages did not demonstrate significant differences $(\mathrm{P}>$ $0,05)$ between the treatments. Hatching values $(\%)$ were significantly higher $(\mathrm{P}<0,05)$ in Woynarovich hatcheries; the survivorship (\%) of the larvae at 48 and 72 hours after hatching were significantly higher $(\mathrm{P}<0,05)$ in Israeli hatcheries; and the percentage of defective larvae was significantly lower $(\mathrm{P}<0,05)$ in Israeli hatcheries in both evaluation times. According to the results, the Israeli hatcheries present better conditions for larvae survivorship.
\end{abstract}

Key words: Larvae culture. Larvae development.

1 Pós-Graduando do Curso de Mestrado em Zootecnia, Área de Concentração Produção Animal, Universidade Federal do Rio Grande do Sul, UFRGS. E-mail: luisbandeirantes@hotmail.com

2 Biólogo Graduado na Universidade Estadual do Norte do Paraná, Campus Cornélio Procópio, UENP. E-mail: jefersondiasdias2008@hotmail

3 Pós-Graduando do Curso de Doutorado em Zootecnia, Universidade Estadual de Maringá, UEM. E-mail: drfornari@hotmail. com

4 Prof. Associado do Departamento de Zootecnia da Universidade Estadual de Maringá, UEM. E-mail: rpribeiro@uem.br

5 Prof. Assistente do Departamento de Ciências Biológicas da Universidade Estadual do Norte do Paraná, UENP,Campus Luiz Meneghel. E-mail: marcoantoniobio@msn.com

* Autor para correspondência 


\section{Introdução}

O pacu (Piaractus mesopotamicus) é uma das espécies de maior relevância dentro do panorama piscícola no Brasil, não só pela qualidade e excelente sabor de sua carne, mas também pelo seu bom crescimento e desempenho em sistemas de cultivo, seu hábito alimentar onívoro, rápido crescimento e fácil adaptação à alimentação artificial (AYALA, 2008).

Bock e Padovani (2000), afirmam que devido à importância, cada vez maior, que vem sendo atribuída à criação de peixes, torna imperativo que os piscicultores aprimorem-se nas técnicas necessárias para assegurar o êxito inicial da criação, como a produção de alevinos para, repovoamento e criação em piscicultura. As técnicas de propagação artificial possibilitam o suprimento de ovos de uma grande variedade de peixes. Além disso, permitem a incubação e a eclosão de ovos e sua criação em condições bem protegidas, independentemente das condições ambientais. Dependendo do sistema, 20 a $70 \%$ dos ovos produzidos têm possibilidade de se transformarem em alevinos, enquanto a taxa de sobrevivência, sob condições naturais, geralmente, é inferior a $1 \%$ dos ovos produzidos (WOYNAROVICH; HORVÁTH, 1983).

Bock e Padovani, (2000) afirmam que quantidade recomendada de ovos de pacus fertilizados por incubadora é de 1000 a 1500 ovos/litro, sendo que um $\mathrm{kg}$ de ovos secos possui em torno de 100 mil ovos (BERNARDINO; ALCÂNTARA; SENHORINI, 1988), já Zaniboni Filho (1992) considera que densidades de 2000 a 2500 ovos/litro podem ser praticadas. Bock e Padovani (2000) relatam que eclosão dá-se de 17 a 19 horas após a fertilização e a absorção do saco vitelínico, o enchimento da bexiga natatória, as aberturas da boca e do ânus, e o movimento natatório horizontal ocorrem entre 100 e 120 horas, isto é, do quarto para o quinto dia, os mesmos autores afirmam ainda que o tempo para que os eventos de desenvolvimento de embriões e larvas supracitados podem variar de acordo com a temperatura da água.

Este trabalho teve por objetivo avaliar a incubação dos ovos, sobrevivência (\%) e a porcentagem de larvas defeituosas de Piaractus mesopotamicus em incubadoras do tipo "Israelense" e "Woynarovich".

\section{Material e Métodos}

O experimento foi conduzido na Estação de Hidrobiologia e Aqüicultura da Duke Energy do Brasil, localizada no município de Salto Grande - SP, de 28 de Fevereiro a 30 de Março de 2009. Utilizou se ovos e larvas obtidos de 10 fêmeas e 20 machos, induzidos com extrato bruto de hipófises de carpa comum (Cyprinus carpio) (EBHC), sendo que as fêmeas receberam doses de $6 \mathrm{mg} \mathrm{EBHC} /$ $\mathrm{kg}$ de peso corporal divididas em duas aplicações espaçadas em 10 horas, onde a primeira aplicação correspondeu $10 \%$ do total da dose e a segunda $90 \%$. Os machos receberam doses únicas de $3 \mathrm{mg}$ $\mathrm{EBHC} / \mathrm{kg}$ de peso corporal concomitantemente a segunda aplicação das fêmeas. Após a extrusão e fertilização um "pool" de ovos hidratados, obtido pela mistura de ovos provenientes de várias fêmeas que responderam ao tratamento hormonal, foi drenado com auxílio de uma peneira e transferido para incubadoras do tipo "Israelense" de 7,5 litros com vazão média de $4 \mathrm{~L} /$ minuto e do tipo "Woynarovich" de 60 litros com vazão média de 1,5 L/minuto. O delineamento experimental foi totalmente casualisado com dois tratamentos e quatro repetições, sendo quatro incubadoras do tipo "Israelense", e quatro incubadoras do tipo "Woynarovich"

A incubadora do tipo "Woynarovich" ou "Funil" geralmente é utilizada para incubação de ovos não aderentes, caracteriza-se por possuir formato cilíndrido-cônico e são construídas em fibra vidro, podendo ser instaladas com o auxílio de suportes de metal. Este tipo de incubadora possui entrada de água em sua base, promovendo um fluxo ascendente que mantém os ovos e larvas em constante movimentação na coluna d'água, 
realizando a renovação constante do volume d'água para eliminar excretas nitrogenadas provenientes do desenvolvimento embrionário e posteriormente das larvas. (WOYNAROVICH; HORVÁTH, 1983; BARROSO; SANTOS, 2006).

A incubadora do tipo "Israelense" é um sistema de incubadoras imersas, descrito por Woynarovich e Horváth (1983). As incubadoras utilizadas neste estudo possuem forma de cone, sendo que suas estruturas de sustentação (base e abertura superior) e superfície de troca de água (tela com espessura 0,5 mm) são construídas em metal inoxidável, as quais ficam mergulhadas em tanques de concreto retangular com volume aproximado de 700 litros e capacidade para oito incubadoras de 7,5 litros cada, fixadas por suportes metálicos. Cada incubadora possui em sua base uma entrada de água independente que distribui o fluxo de forma semelhante a um chuveiro conectada a uma torneira através de mangueiras.

As incubadoras "israelenses" receberam 0,5 litros de ovos hidratados/repetição ou aproximadamente 6100 ovos/L, já as incubadoras "Woynarovich" receberam 1,5 litros de ovos/repetição ou aproximadamente 2300 ovos/L. Para determinação da taxa de fertilização (\%), foram coletadas três amostras/repetição de $2 \mathrm{~mL}$ de ovos após 8 horas do inicio do experimento, com o auxílio de uma peneira e uma proveta de $10 \mathrm{~mL}$, a análise foi realizada em placa de contagem de ovos. A temperatura da água do sistema foi monitorada como o auxílio de um termômetro digital.

Determinou se a taxa de eclosão (\%) após 20 horas a fertilização, quando o procedimento de limpeza das incubadoras para retirada de grumos e matéria orgânica, decorrente da eclosão dos ovos iniciou se, já a sobrevivência (\%) e a porcentagem de larvas defeituosas dos tratamentos foram estimadas após 48 horas e 72 horas da eclosão. Para as três ultimas variáveis citadas coletou se 3 amostras de $25 \mathrm{~mL} /$ repetição com a ajuda de um becker. A análise das larvas foi realizada através de contagem com pipetas de Pasteur de $25 \mathrm{~mL}$. Para o diagnostico de larvas defeituosas utilizou se um microscópio estereoscópico com aumento de 10 vezes, considerou se defeituosas larvas que apresentavam deformidades no corpo ou natação errática (OKAMOTO, 2004). Antes de cada coleta tomou se o cuidado de homogeneizar o conteúdo das incubadoras com o auxílio de uma haste de vidro. Os resultados foram submetidos à análise de variância realizada pelo software Sistema para Análise e Separação de médias em experimentos Agrícolas Versão 8.2 (CANTERI et al., 2001). O teste de Tukey foi empregado, a significância de $5 \%$.

\section{Resultados e Discussão}

A temperatura média da água do sistema no período do experimento foi $28,4^{\circ} \mathrm{C}$, portanto dentro dos padrões de temperatura recomendado para o desenvolvimento de espécies de peixes tropicais, de acordo Baldisserotto (2002).

Os valores médios de taxa de fertilização $(\mathrm{P}>0,05)$ não diferiram entre os tratamentos (Tabela 1), já que foi utilizado um "pool" de ovos obtidos de varias fêmeas, distribuído entre os diferentes tratamentos. De acordo com Bernardino, Alcântara e Senhorini (1988) estes resultados estão dentro dos padrões aceitáveis para a propagação artificial da espécie em questão.

Dumont-Neto et al. (1997) para avaliar a reprodução de Salminus brasiliensis na estação de pesquisa de Volta - MG, utilizaram incubadoras do tipo "funil “ de volume de 20,60 e 200 litros, com densidade média de 1000 ovos/litro. Nestas condições obtiveram taxas de fertilização médias sem diferenças significativas entre $o$ três tipos de incubadoras, estes resultados são semelhantes aos obtidos no presente estudo. Sato et al. (2006) avaliando a reprodução induzida de Astynax bimaculatus e Tetragonopterus chalceus, empregaram incubadoras do tipo "funil" de 20 litros com vasão média de 0,75 litros/mim e desindade de 
20 gramas de ovos/litro, alcançaram taxas médias de fertilização acima de 70 \%. Os autores afirmaram que o sistema incubadora utilizado mostrou se eficiente na hidratação dos ovos e na eficiência de incubação não interferindo na taxa de fertilização, já que as espécies do estudo apresentam ovos levemente adesivos. Diante destes resultados pode se afirmar que as incubadoras avaliadas neste experimento não interferiram na hidratação, incubação e consequentemente na fertilização dos ovos

A taxa de eclosão média(\%) foi significativamente maior nas incubadoras do tipo Woynarovich (54,72\%). Zaniboni Filho (1992) avaliando diferentes densidades de ovos de Colossoma macropomun em incubadoras do tipo "funil" ou "Woynarovich" de 20 e 60 litros obteve resultados semelhantes ao presente estudo de taxa de eclosão em $13 \%$ das incubadoras que utilizaram densidades de ovos inferiores a 2500 ovos/litro. O mesmo autor ainda relata que nesta densidade de estocagem de ovos, houve a necessidade de nas primeiras 35 a 40 horas de incubação, realizar uma limpeza das incubadoras para retirada de grumos de matéria orgânica, formadas pelo depósito de ovos e cascas de ovos, que se depositavam na tela reduzindo a área de drenagem da água. Já quando a densidade de estocagem de ovos foi superior a 2500 ovos/litros foram necessárias realizar duas ou mais limpezas, até o final do período de eclosão dos ovos.

Cerqueira, Mioso e Canarin (2005), em estudos de reprodução induzida de Centropomus parallelus, avaliaram a utilização de incubadoras do tipos "funil" de 37 litros com diferentes densidades (ovos/l) e renovação continua de 4,5 L/mim, sob estas condições obtiveram taxa de eclosão média de $52 \%$, portanto resultado semelhante ao alcançados neste trabalho. Os autores afirmaram também que as taxas de eclosão obtidas foram de certo modo elevadas, uma vez que a taxa de fertilização não foi considerada para seu cálculo e os resultados parecem igualmente indicar que a qualidade da água foi mantida ao longo do processo. Concluiram ainda que o sistema de incubação funcionou adequadamente e provavelmente abaixo de sua capacidade máxima de suporte, pois permitiu o uso de densidades elevadas de ovos (acima de 1000 ovos/L) com considerável eficácia, estando provavelmente associada ao fluxo contínuo de água de cada incubadora.

Barroso e Santos (2006) avaliando um novo sistema de incubação de ovos de peixes de água doce com sistema de filtragem de água acoplado comparado ao sistema tradicional, (incubadoras do tipo "funil"), obteve para ovos de Colosoma macropomum e Prochilodus lineatus taxas de eclosão acima de 90\% nos dois sistemas avaliados, sem diferenças significativas. Diante destes resultados o autor conclui que os sistemas de incubação utilizados não interferiram na taxa de eclosão. Este resultado é contrastante ao obtido no presente estudo, onde as taxas de eclosão média dos sistemas avaliados diferiram significativamente com baixo coeficiente de variação.

As médias de sobrevivência (\%) das larvas após 48 horas da eclosão foram significativamente maior nas incubadoras do tipo Israelense, média de: 97,94\%. O mesmo foi observado após 72 horas da eclosão, onde as incubadoras do tipo Israelense apresentaram média de: 94,96\%. Os resultados de sobrevivência de larvas nos dois tipos de incubadoras avaliadas neste trabalho são semelhantes aos obtidos por Bernardino, Alcântara e Senhorini (1988) em estudos de propagação artificial de Piaractus mesopotamicus. Já Barroso e Santos (2006) trabalhando com sobrevivência de larvas de Colosoma macropomum e Prochilodus lineatus em diferentes sistemas de incubação obtiveram resultados inferiores aos alcançados no presente estudo em incubadoras do tipo "funil". Os autores atribuiram os resultados, a direção do fluxo da água no interior deste tipo de incubadora e ao formato da mesma, o que favorece o choque das larvas com as laterais da incubadora, podendo causar mortalidade das larvas.

A porcentagem de larvas defeituosas foi 
significativamente menor nas incubadoras do tipo Israelense nos dois períodos avaliados, 6,91\% após 48 horas a eclosão e 7,79\% 72 horas após a eclosão. Woynarovich (1986) afirma que as principais causas para deformidades em embriões e larvas de peixes são a insuficiência de oxigênio durante o cultivo e a carência de nutrientes essenciais na dieta dos reprodutores o que afeta a composição do vitelo quantitativamente e qualitativamente. $\mathrm{O}$ presente estudo não mensurou a oxigenação da água nos dois tipos de incubadoras avaliadas, assim sugere se a repetição deste experimento, para avaliar os parâmetros físico químicos e a influencia destes na incubação de ovos e sobrevivência de larvas. $\mathrm{O}$ autor supracitado afirma ainda que de 3 a $10 \%$ de embriões e larvas deformados podem ser encontrado em cada lote de ovos incubados, esta afirmação condiz com os resultados de larvas defeituosas obtidos neste estudo.

Tabela 1. Valores Médios de (\%) fertilização, (\%) eclosão, (\%) sobrevivência após 48 h e $72 \mathrm{~h}$ à eclosão e (\%) larvas defeituosas após udo48h e 72 h à eclosão. Desvio Padrão ( \pm ).

\begin{tabular}{lccc}
\hline Variáveis & Woynarovich & Israelense & C.V* (\%) \\
\hline (\%) Fertilização & $64,78^{\mathrm{A}} \pm 1,9$ & $67,03^{\mathrm{A}} \pm 6,4$ & 7,21 \\
$(\%)$ Eclosão & $54,72^{\mathrm{A}} \pm 0,47$ & $51,67^{\mathrm{B}} \pm 0,82$ & 1,26 \\
$(\%)$ Sobrevivência após 48 h à eclosão & $82,64^{\mathrm{B}} \pm 3,0$ & $97,94^{\mathrm{A}} \pm 6,7$ & 5,87 \\
$(\%)$ Sobrevivência após 72 h à eclosão & $69,46^{\mathrm{B}} \pm 11,12$ & $94,96^{\mathrm{A}} \pm 2,65$ & 10,14 \\
$(\%)$ Larvas defeituosas após 48 h à eclosão & $13,65^{\mathrm{A}} \pm 6,8$ & $6,91^{\mathrm{B}} \pm 3,1$ & 27,23 \\
$(\%)$ Larvas defeituosas após 72 h à eclosão & $15,16^{\mathrm{A}} \pm 3,4$ & $7,79^{\mathrm{B}} \pm 2,6$ & 26,64 \\
\hline
\end{tabular}

Médias na linha com a mesma letra não possuem diferenças significativas $(P \geq 0,05)$.

Coeficiente de variação* (C.V \%)

\section{Conclusão}

De acordo com os resultados obtidos, as incubadoras do tipo "Israelense" demonstraram melhores condições para a sobrevivência das larvas de pacu quando comparadas com as incubadoras do tipo "Woynarovich", devido à ausência de estudos desenvolvidos com incubadoras do tipo "Israelense" sugere se a realização de experimentos que avaliem a sua utilização na incubação de ovos das diferentes espécies de peixes, assim como sua viabilidade econômica.

\section{Agradecimentos}

À Duke energy do Brasil por ceder toda estrutura física de sua estação de hidrobiologia e aqüicultura e material biológico sem os quais o presente estudo não poderia ser realizado.

\section{Referências}

AYALA, J. A. C. Ontogenia do sistema sensorial de Pacu Piaractus mesopotamicus, (Holmberg, 1887) (Characidae: serrasalmidae). 2008. Dissertação (Mestrado em Aqüicultura) - Universidade Estadual Paulista, Centro de Aqüicultura. Jaboticabal.

BALDISSEROTTO, B. Fisiologia de peixes aplicada piscicultura. Santa Maria: UFSM, 2002. 212 p.

BARROSO, H. G.; SANTOS A. J. G. Incubadora HB para ovos de peixes de água doce e sua larvicultura (Patente: MU 7903279-6*). Revista Brasileira de Engenharia de Pesca, São Luís, MA, v. 1, n. 1, p. 119-143, 2006.

BERNARDINO, G.; ALCÂNTARA, R. C. G. de; SENHORINI, J. A. Procedimentos para reprodução 
induzida e alivinagem do tambaqui Colossoma macropomun e pacu Piaractus mesopotamicus. In: TRABALHO APRESENTADO NO SIMPÓSIO LATINO AMERICANO, 6., 1988, Florianópolis. Anais... Florianópolis: ABRAq/ALA, 1988. p. 193.

BOCK, C. L.; PADOVANI, C. R. Considerações sobre a reprodução artificial e alevinagem de pacu (Piaractus mesopotamicus, Holmberg, 1887) em viveiros. Acta Scientiarum, Maringá, v. 22, n. 2, p. 495-501, 2000.

CANTERI, M. G.; ALTHAUS, R. A.; VIRGENS FILHO, J. S.; GIGLIOTI, E. A.; GODOY, C. V. SASM - Agri: sistema para análise e separação de médias em experimentos agrícolas pelos métodos Scoft - Knott, Tukey e Duncan. Revista Brasileira de Agrocomputação, Ponta Grossa, v. 1, n. 2, p. 18-24. 2001.

CERQUEIRA, V. R.; MIOSO, R.; CANARIN, M. Indução de desova com fertilização artificial e natural e incubação de ovos de robalo fecha (Centropomus parallelus). Revista Atlântica, Rio Grande, v. 27, n. 1, p. 31-38, 2005.

DUMONT-NETO, R.; PELLI, A.; FREITAS, R. O.; COSTA, C. L. da; BARBOSA, N. D. de C.. Reprodução induzida do dourado (Salminus maxillosus, VALENCIENNES, 1849) na estação de pesquisa e desenvolvimento ambiental de Volta-Grande CEMIG/ EPDA/V.G. Revista Unimar, Maringá, v. 19, n. 2, p. 439445, 1997.

OKAMOTO, M. H. Efeito da temperatura sobre ovos e larvas do linguado Paralichthys orbignyanus. 2004. Dissertação (Mestrado em Aquicultura ) - Universidade Federal do Rio Grande, Rio Grande.

SATO, Y.; SAMPAIO, E. V.; FENERICH-VERANI, N.; VERANI, J. R. Biologia reprodutiva e reprodução induzida de duas espécies de characidae (Ostheichytheis, characiformes) da bacia do rio São Francisco, Minas Gerais, Brasil. Revista Brasileira de Zoologia, Curitiba, v. 23, n. 1, p. 267-273, 2006.

WOYNAROVICH, E. Tambaqui e pirapitinga: propagação artificial e criação de alevinos. Brasília: CODEVASF, Companhia de Desenvolvimento do Vale do São Francisco, 1986. 42 p.

WOYNAROVICH, E.; HORVÁTH, L. A propagação artificial de peixes de águas tropicais: manual de extenção. CHAMA, V. L. M. (Trad.). Brasília: FAO/ CODEVASF/CNPQ, 1983. p. 225.

ZANIBONI FILHO, E. Incubação, larvicultura $e$ alevinagem do tambaqui (Colossoma macropomum CUVIER, 1818). 1992. Tese (Doutorado em Ecologia e Recursos Naturais) - Centro de Ciências Biológicas e da Saúde. Universidade Federal de São Carlos, São Paulo. 